\title{
ANALYSIS OF LAND SURFACE DEFORMATION GRADIENT BY DINSAR
}

\author{
Karima Hadj-Rabah ${ }^{1}$, Faiza Hocine ${ }^{2}$,SawsenBelhadj-Aissa ${ }^{3}$ and \\ Aichouche Belhadj-Aissa $^{4}$ \\ ${ }^{1,2,3,4}$ Department of Telecommunications,University of Sciences and \\ Technology Houari Boumediene (USTHB), Algiers, Algeria
}

\begin{abstract}
The progressive development of Synthetic Aperture Radar (SAR) systems diversify the exploitation of the generated images by these systems in different applications of geoscience. Detection and monitoring surface deformations, procreated by various phenomena had benefited from this evolution and had been realized by interferometry (InSAR) and differential interferometry (DInSAR) techniques. Nevertheless, spatial and temporal decorrelations of the interferometric couples used, limit strongly the precision of analysis results by these techniques. In this context, we propose, in this work, a methodological approach of surface deformation detection and analysis by differential interferograms to show the limits of this technique according to noise quality and level. The detectability model is generated from the deformation signatures, by simulating a linear fault merged to the images couples of ERS1 / ERS2 sensors acquired in a region of the Algerian south.
\end{abstract}

\section{KEYWORDS}

Radar Interferometry (InSAR \& DInSAR), Surface Deformation Gradient (SDG), Coherence, Resolution, Filtering

\section{INTRODUCTION}

The interferometry (InSAR) is a technique allowing the generation of altimetric information and its variations from couples of SAR radar images. Although, interferometric techniques had known an important development in terms of correction treatments and telemetric phase analysis approaches and methods, their evaluation in comparison with the required precision by geodesy, showed limitations as for their use in cartography deformation and sources characterization causing these last. The quality of interferometric products depends, from one side, on the acquisition geometry and periodicity of radar systems, and from the other side on, the atmospheric conditions, the scene degradations and the surface state observed at a moment ' $t$ '. As a result, differential interferometry (DInSAR) processes do not allow in all cases the deformations detection. However, it is essential to know the deformations that can be detected by the differential interferometry, thus making it possible to decide on the best solution to opt for measuring and monitoring a surface displacement phenomenon [1][2].

Massonnet and Feigl [3] had shown that the necessary condition for the detection of a deformation is that the maximum surface deformation gradient (SDG) equals a fringe by the pixel resolution. Indeed, a fringe of deformation equals half a wavelength. This definition depends on two parameters: the wavelength and the resolution, these last are fixed and specified for each

Dhinaharan Nagamalai et al. (Eds) : ACSIT, ICITE, SIPM - 2018

pp. 127-136, 2018. (C) CS \& IT-CSCP 2018

DOI : $10.5121 /$ csit.2018.80810 
sensor. However, in reference to this relation, SAR radars can detect several types of variable intensity deformations. Unfortunately, in practice, the interferometric measurements are too noisy. These phenomena of noise and phase discontinuity are essentially due to: spatial and temporal decorrelation, atmospheric heterogeneity and others [4][5]. Therefore, the small and the large deformations become undetectable when the level of noise is high.

Otherwise, the estimation of the maximum surface deformation gradient (SDG) does not take into account the noise factor [6] where the first indicator is the interferometric coherence. In practice, the lower the coherence is, the more the maximum SDG decreases [7]. In this context, the work that we are about to present is a contribution to the modelling of the surface deformation gradient from the differentials interferograms. To show the effectiveness of this modelling, we proceeded at first by simulating a brittle deformation (linear fault) which we had integrated in pairs of the ERS1/ERS2 sensor images. The remainder of the paper is organized as follows: a synthesis on the deformation detectability models by DInSAR will be presented in section 2, section 3 will include a description of the methodological approach that we propose. Section 4 will be devoted to the deformation gradient modeling as well as the obtained results. To finish, we generalize in section 5 our modelling for a case of a real deformation. The paper will end with a general conclusion and perspectives in order to enrich this work.

\section{RELATED WORK}

The first formula based on the consideration of Massonet and Feigl [3] defines the maximum deformation gradient as:

$$
D_{m}=\varphi_{2 \pi}^{d i f} * \frac{1}{r_{g}}
$$

Where: $\varphi_{2 \pi}^{\text {dif }}$ is the differential phase of one fringe (a turn of $2 \pi$ ) which corresponds to half the wavelength $\lambda$ and the pixel resolution $r_{g}$.

In order to introduce the coherence which best describes the noise effect, Baran et al. [6] have proposed a functional model to determine the minimum and the maximum surface deformation gradient for different values of the coherence, by using real images with a simulated deformation (surface subsidence) for a number of looks L equals 5. Subsequently, Jiang and Li [8] have extended this model in order to adapt it for different number of looks $(\mathrm{L}=1,5$, and 20). In the same context, Wang and Li [9] had resumed the same work for the acquired data by the PALSAR sensor. Recently, Hadj-rabah and Hocine [10] had proposed an automatic approach for surface deformation detection based on a multi-scales analysis that can be adapted and implemented to determine the minimum and the maximum surface deformation gradient instead of the subjective approach implemented in the works mentioned above. These works are focused on a single type of deformation (mining subsidence) and do not include a spatial filtering step in advance. For our part, we proposed a methodological approach (see Fig. 1) for surface deformation gradient detection and analysis for different resolutions and noise levels. In addition, we have analyzed the deformation detectability for a linear fault.

\section{METHODOLOGICAL APPROACH}

In order to achieve our goal,A linear fault of the surface is modeled by simulating deformation images with different parameters representing its temporal evolution (see Fig. 2). These deformations are then wrapped (converted) into phase images. They have been merged with Single Look Complex (SLC) images considered as the 'master' images giving birth to the modified SLC images containing the deformation signature. The generating process of differential interferograms is, then applied. In the course of this procedure, a step of spatial 
filtering is performed and the resolution is gradually changed in order to observe the effect of noise as well as the level of detail on the deformations detectability. the coherence $\gamma$ and the deformation gradient are calculated for each generated differential interferogram.

Finally, the modeling is carried out basically, on a step of decision. This last consists in deciding if the deformation is detectable or not, interactively.

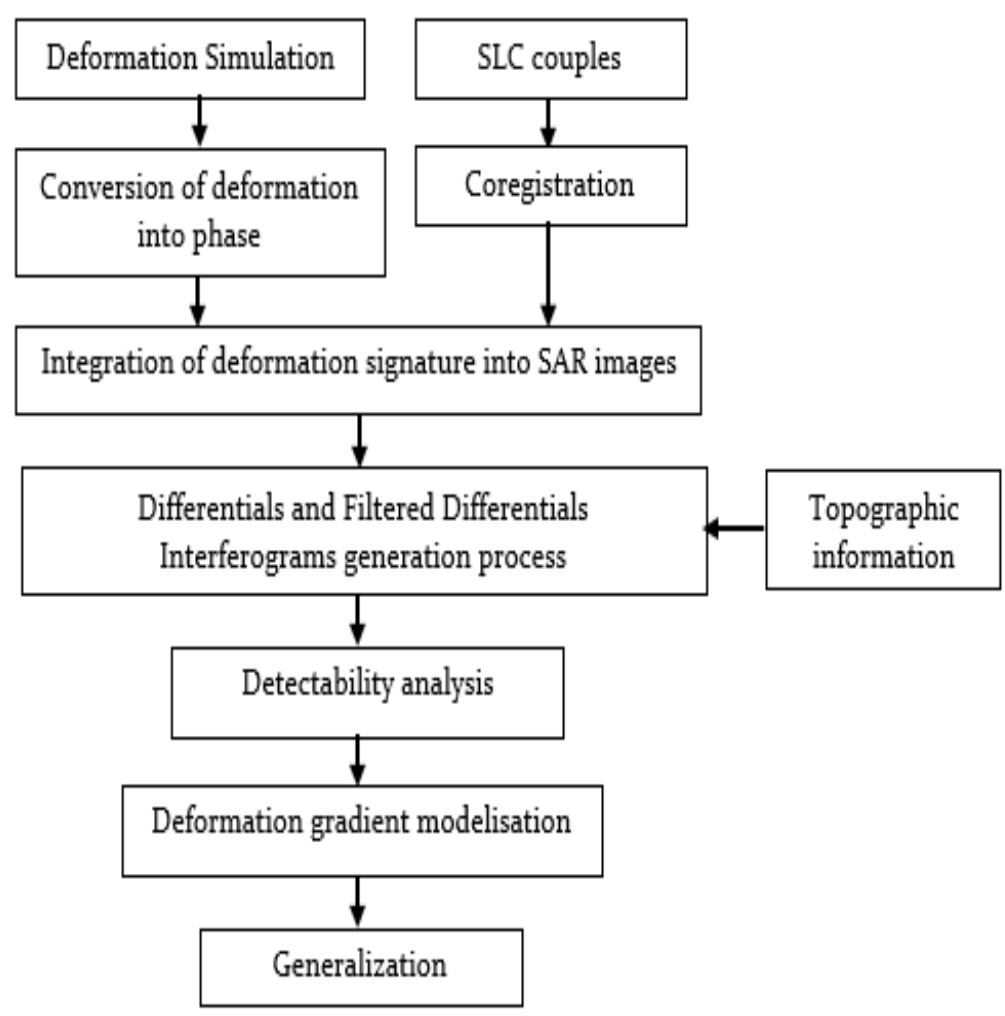

Figure 1. Proposed approach

\subsection{Deformation Simulation}

The simulated deformation according to a direction, supposed to be the acquisition one, is gendered by a linear fault. The spatial two-dimensional function $f$ (following azimuth and distance directions) has been adopted to model such a deformation with a scaling obtained by varying the parameter ' $h$ ', also called the deformation temporal amplitude. Its expression is as follows:

$$
f(R, A, h)=h *[A * \cos \theta+R * \sin \theta]
$$

With: $R$ and $A$, the coordinates of the deformation image following the range and the azimuthal directions respectively. The value of the angle $\theta$ representing the fracture line orientation is considered constant, assuming that the deformation coincides with the radar sight angle, thus we obtain a series of simulated images, which their 3D representation shows the deformation evolution as a function of time (see Fig. 2). 

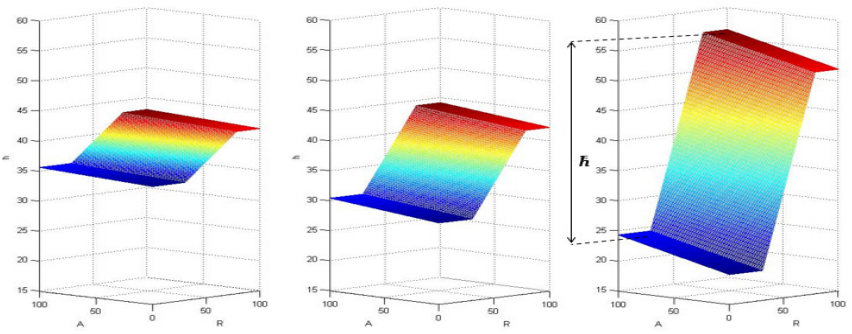

Figure 2. Simulated deformation in 3D

The deformation parameters in the simulated images are represented in the following table:

Table 1. Parameters of simulateddeformation.

\begin{tabular}{|l|l|l|l|}
\hline \multirow{2}{*}{ Name } & Simulated Surface Deformation & \multicolumn{3}{|l|}{} \\
\cline { 2 - 4 } & SD1 & SD2 & SD3 \\
\hline $\boldsymbol{\theta}$ & $35^{\circ}$ & 0.084 & 0.14 \\
\hline $\mathbf{h}(\mathbf{m})$ & 0.014 & \multicolumn{2}{|l}{} \\
\hline
\end{tabular}

\subsection{Deformation Signature Integration}

The variation of the parameter ' $\mathrm{h}$ : fault depth' makes it possible to generate three deformation forms at surface, noted SD1, SD2 and SD3. Surface fault signatures are then generated by merging the deformation to one of the two SLC images of the interferometric pairs. The interferograms are generated from the modified images. The selected SLC images have different coherences and resolutions values ( $8 \mathrm{~m}, 20 \mathrm{~m}$ and $40 \mathrm{~m}$ respectively). A filtering step is applied on the complex interferograms consisting to attenuate the noise effect mainly due to spatial decorrelation, geometry effects of acquisition and observed field. Interferometric pretreatments are applied, namely: orbital fringes removal, topographic pair generation from a digital elevation model (DEM) of the region by bringing it back to interferometric pairs geometry.

\subsection{Detectability Analysis}

The deformation fringes in the differential interferograms are analyzed by an interactive method based on rows and columns profile plots. Three groups of interferograms are represented in figures 3 to 6 , in order to illustrate the necessary fringes analysis for detectability decision, for different coherences $\gamma$, and resolutions values as well as filtering level.
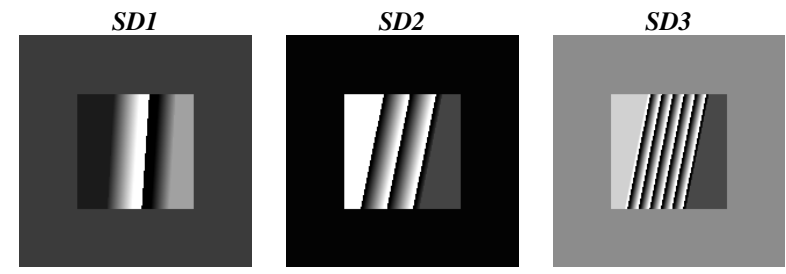

Figure 3. Interferometric phase of the simulated deformations

Fig. 3 and Fig. 4 show the interferometric phase of the simulated deformations (SD1 to SD3) and its corresponding differentials interferograms, with different coherence values for a $20 \mathrm{~m}$ resolution. 

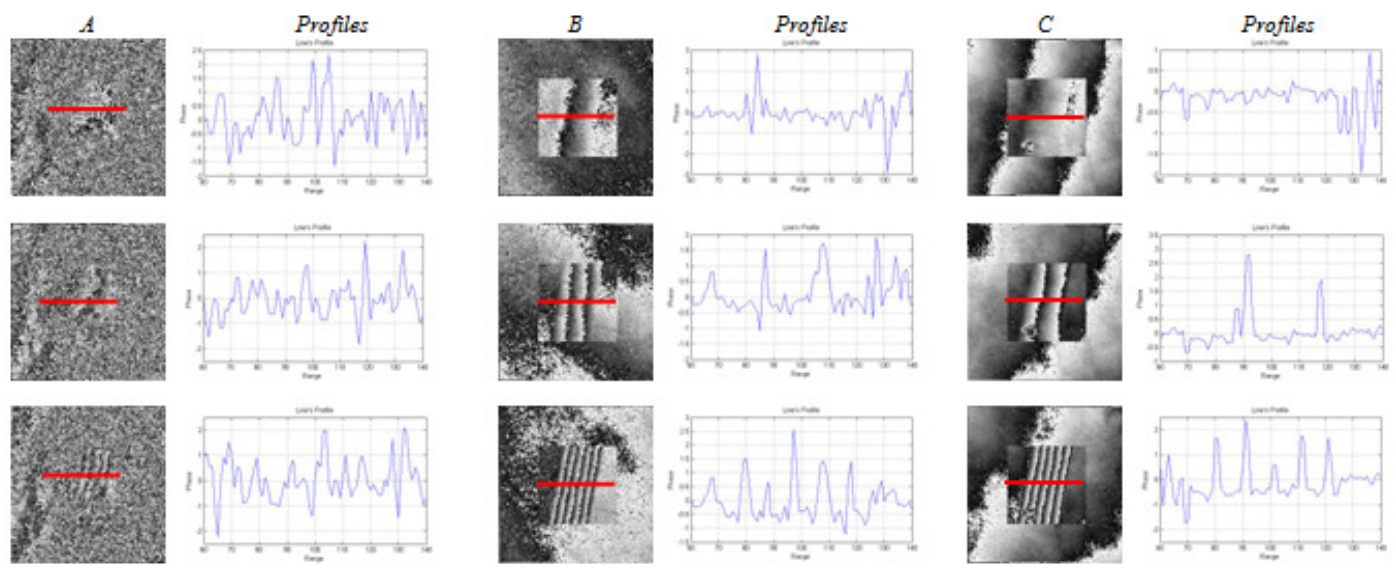

Figure 4. Differentials interferograms and their profiles (red lines), corresponding to simulated deformations SD1 to SD3 with different coherence values $(a) \gamma_{\mathrm{A}}=0.582355,(b) \gamma_{\mathrm{B}}=0.679226$ and $(c) \gamma_{\mathrm{C}}=$ 0.778999 , for a resolution of $20 \mathrm{~m}$

These results show that the more the coherence is, the best the deformation fringes are distinguished and detected. For low coherence values, no deformation can be detected properly, (e.g., image A). On the other hand, for a same coherence value, the more the deformation gradient is, the more the deformation fringes are distinguished and detected.

Fig. 5 shows the differentials interferograms corresponding to deformations SD1 to SD3 with and without filtering for a $20 \mathrm{~m}$ resolution. These results show that the noise spatial filtering makes the fringes contours more readable, thus facilitating the detectability analysis.

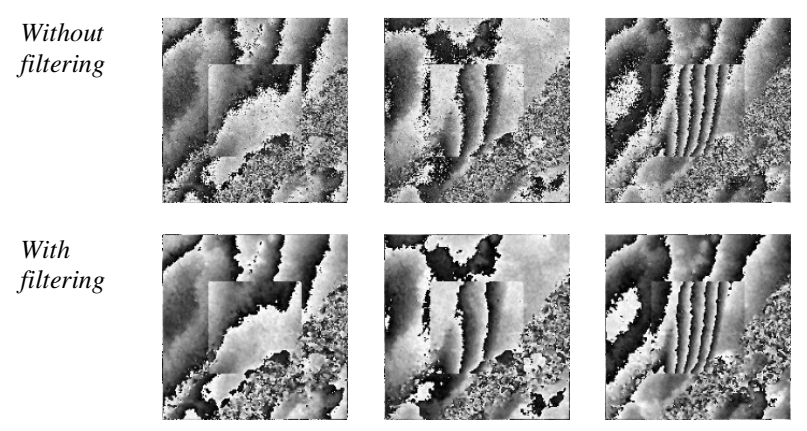

Figure 5. Differentials interferograms corresponding to simulated deformation SD1 to SD3 with a coherence equals to $\gamma_{D}=0.697129$, for a resolution of $20 \mathrm{~m}$ with and without filtering

Regarding the resolution, we have noticed from the results shown in Fig. 6, that the differentials interferograms using a $40 \mathrm{~m}$ resolution are more smoothed than those using an $8 \mathrm{~m}$ resolution. However, if the deformation is very deep, the number of fringes increases and their width decreases, decreasing the resolution leads to a fringes elimination and an erroneous estimation of the deformation. In the same analysis sense, we have noticed that the simulated images for which the deformation fringes are more tighter and the deformation gradient is big (e.g., SD3), are better observed in the case of interferograms using a $20 \mathrm{~m}$ resolution. This result presents a better compromise between the use of a $40 \mathrm{~m}$ and $8 \mathrm{~m}$ resolutions. 
(a)

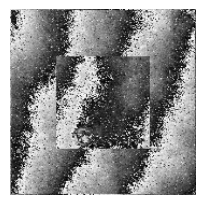

(b)

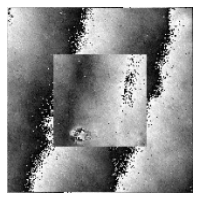

(c)

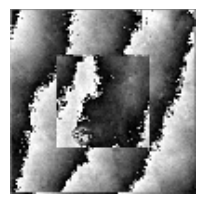

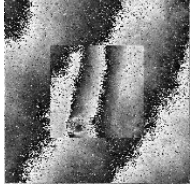
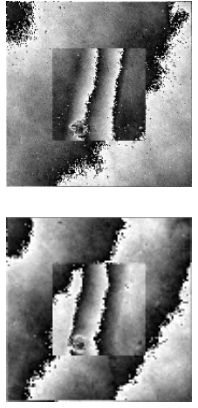
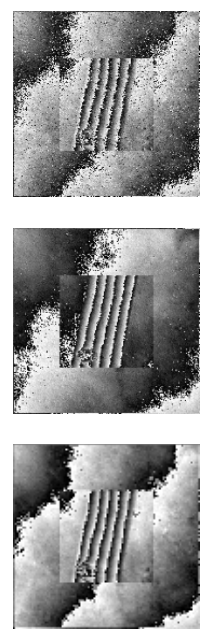

Figure 6. Differentials interferograms corresponding to simulated deformation SD1 to SD3 and a coherence equals to $\gamma_{C}=0.778999$, with different resolutions: (a) $8 \mathrm{~m}$, (b) $20 \mathrm{~m}$ and (c) $40 \mathrm{~m}$

\section{Surface Deformation Gradient Modelling by DInSAR}

The objective of DInSAR deformation detectability analysis is establishing two deformation gradient equations in function of the coherence (see Fig. 7). The linear curves, obtained from the two equations, allow the delimitation of detectability surface in function of the DInSAR quality product. The intersection, the slopes and the offset of the two curves define the deformation detectability model with respect to types of SAR radar images set to be explored in monitoring and mapping applications of land surfaces and/or Over-surfaces movements.
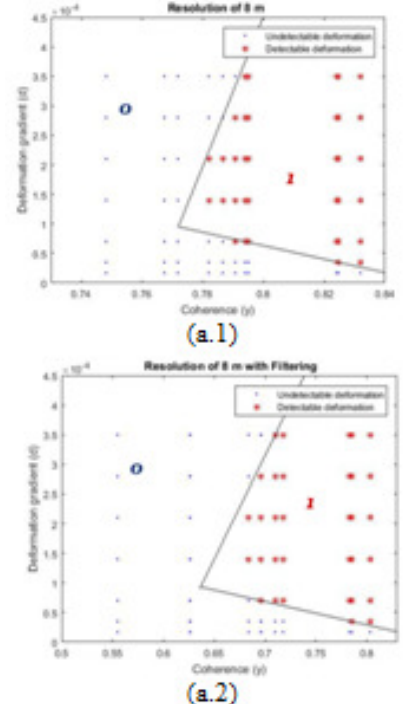

(a.2)

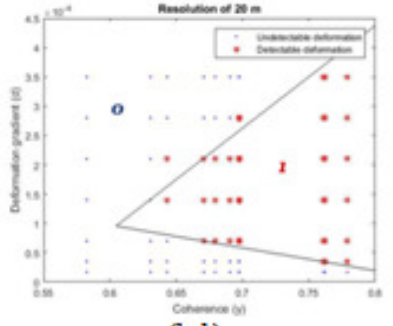

(b.1)

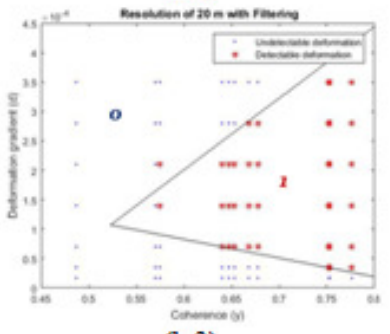

(b.2)

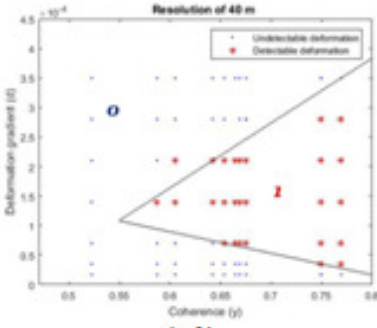

(c.1)

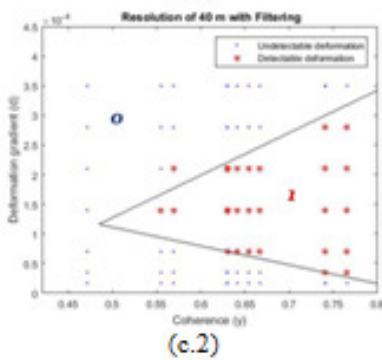

Figure 7. Observations and linear models $d_{\min }(\gamma)$ and $d_{\max }(\gamma)$, for a resolution (a) $8 \mathrm{~m},(\mathrm{~b}) 20 \mathrm{~m}$ and (c) 40 $\mathrm{m},(1)$ without and (2) with spatial filtering 


\subsection{Model Parameters}

This model is defined by two parameters: the coherence and the deformation gradient [6]. The coherence describes the correlation degree between the two SLC images, the modified master image MS and slave image $S$; it is given by:

$$
\gamma=\frac{\left|\sum_{i=1}^{L} M S_{i} \cdot S_{i}{ }^{*}\right|}{\sqrt{\sum_{i=1}^{L}\left|M S_{i}\right|^{2} \sum_{i=1}^{L}\left|S_{i}\right|^{2}}}
$$

Where: $L$ is the number of looks corresponding to its appropriate resolution value.

The deformation gradient $d$ is defined as:

$$
d=\frac{D A}{r}
$$

Where: $D A$ is the deformation amplitude and ris the images resolution according to the range direction.

\subsection{Interactive Model Analysis}

Each differential interferogram, obtained for a given coherence threshold and a given resolution, associated with the $2 \mathrm{D}$ profiles plots are visually analyzed, the decision making on the detectability is related to the fringes limits perception: discernable fringes. On a $d=f(\gamma)$ graph, we attribute a value ' 1 ' if it is discernible, otherwise the value is equal to zero. Fig. 7 represents the deformation gradients $(d)$ plots as a function of the coherence $(\gamma)$, for the different resolution values $(8 \mathrm{~m}, 20 \mathrm{~m}$ and $40 \mathrm{~m})$, with and without the spatial filtering application. By observing the six graphs, the clouds of points forming the decision that equals 1 , represented by the red stars can be delimited by two linear curves (one upper bound and another lower one) [6][8]. The minimum and the maximum SDG differ from one resolution to another, and for the same resolution, the spatial filtering makes it different. As the resolution decreases, the SDG upper bound becomes lower; the same happens for the minimum SDG, this means that the Max(SDG) and the $\operatorname{Min}(\mathrm{SDG})$ vary in the same direction as the resolution.

Linear plots are proposed to approximate the SDG lower and upper boundaries. For this purpose, we obtained a deformation gradient modelling with a resolution of $8 \mathrm{~m}, 20 \mathrm{~m}$ and $40 \mathrm{~m}$ respectively, which their equations are the following (with a normalization factor of $\left(10^{-4}\right)$ ):

- Without spatial filtering

$$
\begin{gathered}
\left\{\begin{array}{c}
d_{\text {min }}=(9,7504-11,4 \gamma) \\
d_{\text {max }}=(-97,241+127,2 \gamma)
\end{array}\right. \\
\left\{\begin{array}{l}
d_{\text {min }}=(3,3064-3,89 \gamma) \\
d_{\text {max }}=(-9,625+17,5 \gamma)
\end{array}\right. \\
\left\{\begin{array}{c}
d_{\text {min }}=(3,085-3,6496 \gamma) \\
d_{\text {max }}=(-4,954+10,989 \gamma)
\end{array}\right.
\end{gathered}
$$

- With spatial filtering

$$
\left\{\begin{array}{c}
d_{\min }=(3,427-3,919 \gamma) \\
d_{\max }=(-21,35+35 \gamma)
\end{array}\right.
$$




$$
\begin{gathered}
\left\{\begin{array}{c}
d_{\text {min }}=(2,735-3,18 \gamma) \\
d_{\text {max }}=(-5,293+12,17 \gamma)
\end{array}\right. \\
\left\{\begin{array}{l}
d_{\text {min }}=(2,699-3,1731 \gamma) \\
d_{\text {max }}=(-2,307+7,162 \gamma)
\end{array}\right.
\end{gathered}
$$

On the other hand, each graph illustrates two areas " 1 " and " 0 ", which cover the surface where the coherence and the SDG value designate the deformations that can or not be detected by DInSAR. The equation below defines the condition of detectability:

$$
\left\{\begin{array}{c}
d_{\min } \leq d \leq d_{\max }=>\text { Area } " 1 " \\
d \leq d_{\min } \text { or } d \geq d_{\max }=>\text { Area } " 0 "
\end{array}\right.
$$

This study shows the usefulness of the approach that we propose during the choice of the technique to be implemented for a good detection of the surface deformation. The establishment of the equation (8) will make it possible to decide if a deformation (linear fault) is detectable or not by differential interferometry, taking into account the noise level in the differential interferogram and by calculating only two parameters: the coherence and the gradient, that describe the noise level and the deformation depth respectively.

\subsection{Discussion}

The proposed methodological approach is based on both simulated and real data, the advantage of using simulated images is to be able to control the dimensions of the deformation. On the other hand, introducing the deformation signature into SLC phase images allows reducing the chances of obtaining interferograms with hidden information of the deformation caused by noise. By comparing the deformation image and the resulting differentials interferograms, with the profiles plots, a set of observations has led us to the establishment of a deformation modelling. In practice, this last is useful to evaluate the capacity of the differential interferometry to detect a surface deformation and to monitor its evolution. However, this model has essentially two limitations. In the first place, the detectability decision depends on an interactive method, which makes it very subjective. In addition, the upper and lower boundaries of the model are not certain. Otherwise, this model has been created based on the deformations modeled by simulation. Although the surface deformations are simulated in the form of a brittle surface deformation designating a linear fault, the methodological approach can be generalized to other kinds of deformations. However, in other kind of deformations, it is not practical to calculate the deformation gradient using equations (5) to (7). Therefore, it is more appropriate to adapt the same methodology in order to simulate specific deformations.

\section{GeneraliZation}

The objective of the generalization is to validate the results obtained in the previous section by checking the reliability of the relationship established between the coherence and the deformation gradient for real data. Since there are no other methods dealing with the same type of deformation and having the same objective and in order to generalize our proposed approach, we used a differential interferogram of the region of Ouargla (Algeria) containing a brittle surface deformation (see Fig. 8). 


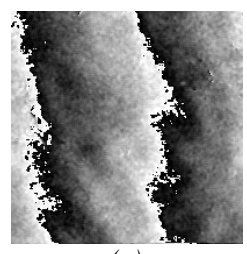

(a)

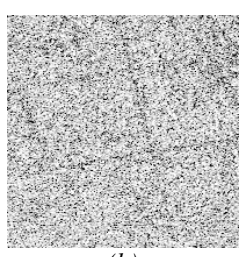

(b)

Figure 8. (a) Real differential interferogram containing a brittle surface deformation and $(b)$ its coherence image

This interferogram was generated for a $20 \mathrm{~m}$ resolution, we have calculated the deformation gradient, as well as the coherence value. Visually, the deformation is detectable, to evaluate the robustness of this modelling, we assessed the found parameters values in order to determine if the point generated belongs actually to the area "1". The results obtained are shown in the following table:

Table 2. Calculated parameters.

\begin{tabular}{|l|l|l|c|}
\hline Gradient & Coherence & $\boldsymbol{d}_{\min }$ & $\boldsymbol{d}_{\max }$ \\
\hline 0.000140 & 0.578806 & $0.8944 \times 10^{-4}$ & $1.7511 \times 10^{-4}$ \\
\hline
\end{tabular}

From the equation (8), we have noticed that the deformation gradient value fills the following condition: $d_{\min } \leq d \leq d_{\max }$, this implies that this deformation is part of the area "1", then it is detectable by radar differential interferometry DInSAR.

\section{CONCLUSIONS}

The objective of this study is to show the contribution and the limits of the differential interferometry in altimetric surfaces and sub-surfaces variations detection and estimation. However, these variations altered by diverse degradation sources contributing to the limitations of the DInSAR and which can be highlighted by an interferometric quality indicator which is the coherence. In this sense, we have analyzed and modeled the surface deformation gradient (SDG) by establishing a relationship between the coherence and the deformation gradient for different resolution values. However, an extension of this work will be possible. Indeed, we have only used a visual detectability analysis for the decision in model construction. Other methods based on an automatic analysis can be adopted. In addition, for a reason of simplicity, linear models were used to separate the regions describing detectable and undetectable deformations. Non-linear models can be envisaged.

\section{REFERENCES}

[1] S.N. Madsen, H. Zebker, \& J. Martin, (1993) "Topographic mapping using radar interferometry: processing techniques", IEEE Transactions on Geoscience and Remote Sensing, Vol. 31, No. 1, pp246-256.

[2] X.L. Ding, G.X. Liu, Z.W. Li, Z.L. Li, \& Y.U. Chen, (2004) “Ground subsidence monitoring Hong Kong with satellite SAR interferometry”, Photogrammetric Engineering and Remote Sensing, Vol. 70, No. 10, pp1151-1156.

[3] D. Massonnet\& K. L. Feigl, (1998) "Radar interferometry and its application to changes in the earth's surface”, Rev. Geophys., Vol. 36, No. 4, pp441-500.

[4] H. A. Zebker\& J. Villasenor, (1992) "Decorrelation in interferometric radar echoes," IEEE Transactions on Geoscience and Remote Sensing, Vol. 30, No. 5, pp 950-959. 
[5] X. L. Ding, Z. W. Li, C. Huang \& Z.R. Zou, (2007) "Atmospheric effects on repeat-pass InSAR Measurement over SHANGAI Region”, J.Atmos. Sol-Terr Phy, Vol. 69, No. 12, pp 1344-1356.

[6] I. Baran, M. Stewart, \&S. Claessens, (2005) "A new functional model for determining minimum and maximum detectable deformation gradient resolved by satellite radar interferometry", IEEE Transactions on Geoscience and Remote Sensing, Vol. 43, No. 4.

[7] S-H. Yun, H. Zebker, P. Segall, A. Hooper, \& M. Poland, (2007) "Interferogram formation in the presence of complex and large deformation”, Geophysical Research Letters, Vol. 34.

[8] Z. W. Li, X. Ding, Z. Jian-Jun, \& G. Feng, (2011) "Modeling minimum and maximum detectable deformation gradients of interferometric SAR measurements", International Journal of Applied Earth Observation and Geoinformation, Vol. 13, No. 5, pp 766-777.

[9] Q. J. Wang, Z. W. Li, Y.N. Du, R. A. Xie, X.Q. Zhang, M. Jiang, \& J-J. Zhu, (2014) "Generalized functional model of maximum and minimum detectable deformation gradient for PALSAR interferometry", Trans. Nonferrous Met. Soc. China, Vol. 24,pp 824-832.

[10] K. Hadj Rabah, F. Hocine, \& A. BelhadjAissa, (2017) “Automatic Detection of Surface Deformations by DInSAR", in Proceedings of 6th International Conference on Telecommunication and Remote Sensing.

\section{AUTHORS}

Karima Hadj-Rabah received the M.Sc. degree in Telecommunication, networks and multimedia from University of Science and technology HouariBoumedienne(USTHB), Algeria, in 2016.She is curently working toward the Ph.D.degree insynthetic aperture radar (SAR)tomography reconstruction using Very High-resolutiondata, in laboratory of image processing and radiation, USTHB, under the supervision of Professor AichoucheBelhadj-Aissa.

Faiza Hocine Graduated from the University of Sciences and Technology Houari Boumedienne (USTHB), Algeria. $\mathrm{PhD}$ in image processing and remote sensing of the same university, in 2015. Currently, she is researcher in Electronics, image processing, remote sensing at USTHB. Her research interests include satellite image processing, SAR interferometry radar.

SawsenBelhadj-Aissa received the M.Sc. degree in Telecommunication, networks and multimedia from University of Science and technology HouariBoumedienne (USTHB), Algeria, in 2013. She is curently working toward the Ph.D. degree in SAR Differential Interferometry: 2D / 3D applied to the detection of surface'sdeformations, in laboratory of image processing and radiation, USTHB, under the supervision of Professor Salah Boughacha.

AichoucheBelhadj-Aissa obtained her engineering degree in electronics from National Polytechnic School, Algiers, the magister degree and the Doctorate in image processing and remote sensing from the University of the Sciences and Technology Houari Boumediene, Algiers. Currently, she is university Professor and head of the research team "GIS and integration of geo-referenced data". The main research themes focus on modeling and analysis of textures and forms, fusion and classification of objects, SAR interferometry-polarimetry and GIS.
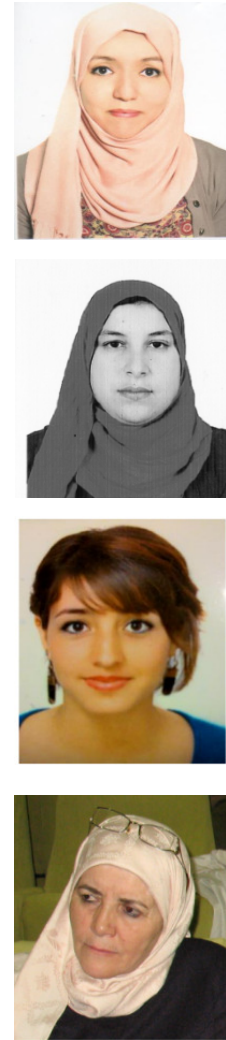